SLAC-PUB-95-7007

September 1995

\title{
RECENT SLAC MEASUREMENTS \\ OF THE SPIN DEPENDENT STRUCTURE FUNCTIONS FOR THE PROTON AND NEUTRON*
}

\author{
GEORDIE ZAPALAC \\ High Energy Physics Dept., University of Wisconsin, 1150 University Ave, \\ Madison, Wisconsin 53706 USA \\ Representing the E142 and E143 Collaborations \\ Stanford Linear Accelerator Center \\ Stanford University, Stanford, California 94309
}

\begin{abstract}
We present results from SLAC experiments E142 and E143 for the spin dependent structure functions of the proton $g_{1}^{p}\left(x, Q^{2}\right)$ and neutron $g_{1}^{n}\left(x, Q^{2}\right)$ measured in deep inelastic scattering of polarized electrons from a polarized target. Experiment E142 measures $\int_{0}^{1} g_{1}^{n}(x) d x=-0.022 \pm 0.011$ at $\left\langle Q^{2}\right\rangle=2(\mathrm{GeV} / \mathrm{c})^{2}$ using a polarized ${ }^{3} \mathrm{He}$ target. Experiment E143 measures $\int_{0}^{1} g_{1}^{p}(x) d x=$ $0.129 \pm 0.011$ at $\left\langle Q^{2}\right\rangle=3(\mathrm{GeV} / \mathrm{c})^{2}$ using a polarized $\mathrm{NH}_{3}$ target. These results are combined at $Q^{2}=3(\mathrm{GeV} / \mathrm{c})^{2}$ to yield $\int_{0}^{1}\left[g_{1}^{p}(x)-g_{1}^{n}(x)\right] d x=0.151 \pm 0.015$. The Bjorken sum rule predicts $0.171 \pm 0.008$.
\end{abstract}

\author{
Presented at the 1994 Meeting of the American Physical Society, \\ Division of Particles and Fields (DPF94) \\ Albuquerque, New Mexico, August 2-6, 1994
}

\section{DISCLAIMER}

\begin{abstract}
This report was prepared as an account of work sponsored by an agency of the United States Government. Neither the United States Government nor any agency thereof, nor any of their employees, makes any warranty, express or implied, or assumes any legal liability or responsibility for the accuracy, completeness, or usefulness of any information, apparatus, product, or process disclosed, or represents that its use would not infringe privately owned rights. Reference herein to any specific commercial product, process, or service by trade name, trademark, manufacturer, or otherwise does not necessarily constitute or imply its endorsement, recommendation, or favoring by the United States Government or any agency thereof. The views and opinions of authors expressed herein do not necessarily state or reflect those of the United States Government or any agency thereof.
\end{abstract}

${ }^{*}$ Work supported in part by U.S. Department of Energy contracts DE-AC03-76SF00515 and DE-AC02-76ER00881. 


\section{INTRODUCTION}

At infinite $Q^{2}$ the spin-dependent structure function $g_{1}(x)$ is interpreted as the charge weighted difference between momentum distributions for quarks with helicities aligned parallel $(\uparrow)$ and anti-parallel $(\downarrow)$ to the helicity of the nucleon:

$$
g_{1}(x)=\frac{1}{2} \sum_{k} e_{k}^{2}\left[q_{k}^{\dagger}(x)-q_{k}^{\downarrow}(x)\right] \equiv \sum_{k} e_{k}^{2} \Delta q_{k}(x)
$$

where $e_{k}$ is the charge of quark species $k$. We adopt the convention that $\int_{0}^{1} \Delta q_{k}(x) d x=\Delta k$ refers to the net helicity of quark species $k=u, d, s$ in the proton. The spin-dependent structure functions for the proton $g_{1}^{p}(x)$ and the neutron $g_{1}^{n}(x)$ are related by the Bjorken sum rule to the axial vector decay constant $g_{A} / g_{V}$ for neutron beta decay. [1] At any experimentally accessible and hence finite $Q^{2}$ the function $g_{1}$ depends on $Q^{2}$ and the Bjorken sum rule must be corrected by perturbative QCD [2]:

$$
\int_{0}^{1}\left(g_{1}^{p}-g_{1}^{n}\right) d x=\frac{1}{6} \frac{g_{A}}{g_{V}}\left[1-\frac{\alpha_{s}\left(Q^{2}\right)}{\pi}-3.58\left(\frac{\alpha_{s}\left(Q^{2}\right)}{\pi}\right)^{2}-20.23\left(\frac{\alpha_{s}\left(Q^{2}\right)}{\pi}\right)^{3}+O\left(\alpha_{s}^{4}\right)\right]
$$

where $\alpha_{s}\left(Q^{2}\right)$ is the QCD coupling constant. The Bjorken sum rule is a consequence of the isospin symmetry of the axial vector current for neutron beta decay; an experimental test of this sum rule is regarded as a test of perturbative QCD.

Neutron beta decay combined with isospin symmetry yields the relation $\Delta u-\Delta d=g_{A} / g_{V}$. The decay $\Sigma^{-} \rightarrow n e^{-} \bar{\nu}$ and the assumption of $\mathrm{SU}(3)$ flavor symmetry yields a second relation $\Delta d-\Delta s=g_{A}^{\Sigma} / g_{V}^{\Sigma}$, where $g_{A}^{\Sigma} / g_{V}^{\Sigma}$ is the axial vector decay constant for $\Sigma^{-} \rightarrow n e^{-} \bar{\nu}$. The measurement $\int_{0}^{1} g_{1}(x) d x$ corrected to the average experimental $Q^{2}$ provides a third equation [3], allowing one to solve for the quark helicities $\Delta u, \Delta d$, and $\Delta s$.

The spin dependent structure function $g_{1}\left(x, Q^{2}\right)$ is measured in deep inelastic scattering by measuring the crosssection asymmetries $A_{\|}=\Delta \sigma_{\|} / \Sigma \sigma_{\|}$and $A_{\perp}=\Delta \sigma_{\perp} / \Sigma \sigma_{\perp}$, where the differences between the cross-sections are $\Delta \sigma_{\|}=\sigma(\uparrow \Uparrow)-\sigma(\downarrow \Uparrow)$, and $\Delta \sigma_{\perp}=\sigma(\uparrow \Rightarrow)-\sigma(\downarrow \Rightarrow)$, and where $\Sigma \sigma$ is the cross-section sum. The symbol $\uparrow$ denotes 


\section{DISCLAIMER}

Portions of this document may be illegible in electronic image products. Images are produced from the best available original document. 
the incoming electron helicity and $\Uparrow(\Rightarrow)$ denotes the longitudinal (transverse) spin of the target nucleon. We have:

$$
g_{1}\left(x, Q^{2}\right)=\frac{F_{1}\left(x, Q^{2}\right)}{D}\left[A_{\|}+\tan (\theta / 2) A_{\perp}\right]
$$

where $\theta$ is the scattering angle, $D=(1-\epsilon)(2-\nu / E) /[\nu / E(1+\epsilon R)], E$ is the incident electron energy, $R\left(x, Q^{2}\right)=$ $\sigma_{L} / \sigma_{T}=\left(1+Q^{2} / \nu^{2}\right) F_{2} / F_{1}-1$ is the ratio of photoabsorption cross-sections for longitudinal and transverse photons, and $\epsilon=\left[1+2\left(1+\nu^{2} / Q^{2}\right) \tan ^{2}(\theta / 2)\right]^{-1}$ is the longitudinal polarization of the virtual photon exchanged in the scattering.

\section{EXPERIMENTS E142 AND E143}

Experiment E142 ran during the fall of 1992 in End Station A (ESA) at SLAC using a gaseous polarized ${ }^{\mathbf{3}} \mathrm{He}$ target and a polarized electron beam at energies of $19.4,22.7$, and $25.5 \mathrm{GeV}$. [4] Data were taken with both longitudinal and transverse target polarizations. Electrons scattered from the target between 6 and $20 \mathrm{GeV}$ were counted in two magnetic spectrometers positioned at scattering angles of $4.5^{\circ}$ and $7^{\circ}$. These conditions allowed a measurement of $g_{1}^{n}$ over the kinematic range $0.033<x<0.6$ and $1<Q^{2}<7.5(\mathrm{GeV} / \mathrm{c})^{2}$.

Experiment E143 ran during the fall of 1993 in ESA using a polarized ${ }^{15} \mathrm{NH}_{3}$ solid target to measure both $g_{1}^{p}$ and $g_{2}^{p}$ and a polarized ${ }^{15} \mathrm{ND}_{3}$ target to measure $g_{1}^{d}$ and $g_{2}^{d}$. Data were taken with longitudinal and transverse target polarizations and with polarized electron beam energies of $9.7,16.2$, and $29.1 \mathrm{GeV}$. Only the preliminary analysis of $g_{1}^{p}$ at $29.1 \mathrm{GeV}$ is reported here. [5] Scattered electrons between 6 and $25 \mathrm{GeV}$ were counted using the same E142 spectrometers with nearly identical detector elements. These conditions correspond to the kinematic range $0.027<x<0.8$ and $1.3<Q^{2}<10(\mathrm{GeV} / \mathrm{c})^{2}$.

\section{A. The E142 Polarized Helium Target}

To first approximation the polarization of the neutron in the ${ }^{3} \mathrm{He}$ nucleus is the same as the polarization of the

${ }^{3} \mathrm{He}$ nucleus. This follows from the assertion that the wavefunction of the ${ }^{3} \mathrm{He}$ nucleus is $87 \%$ in the symmetric $\mathrm{S}$ state with both proton spins aligned antiparallel due to the Pauli exclusion principle. [6] The target was a $30 \mathrm{~cm}$ glass tube filled with ${ }^{3} \mathrm{He}$ to a density of $2.3 \cdot 10^{20}$ atoms $/ \mathrm{cm}^{3}$. The beam entered and exited through $0.012 \mathrm{~cm}$ 
thick glass end windows. Helmholtz coils provided a uniform 30 Gauss magnetic field for the ${ }^{3} \mathrm{He}$ quantization axis. A small amount of optically pumped $\mathrm{Rb}$ vapor polarized the ${ }^{3} \mathrm{He}$ nucleus through the hyperfine interaction during spin-exchange collisions between $\mathrm{Rb}$ and ${ }^{3} \mathrm{He}$ atoms. [7] The target polarization $P_{T}$ was typically $35 \%$ and measured with an uncertainty of $\delta P_{T} / P_{T}=7 \%$ by NMR adiabatic fast passage. The NMR measurement was calibrated by measuring the thermal equilibrium polarization of water in the same magnetic field. Every few hours the target polarization was reversed.

\section{B. The E143 Polarized Ammonia Target}

Radiation doped ${ }^{15} \mathrm{NH}_{3}$ granules were used for the polarized proton target. [8] The $\mathrm{NH}_{3}$ granules were contained in a $3 \mathrm{~cm}$ long permeable target cell submerged in liquid helium and maintained at $1^{\circ} \mathrm{K}$ by a helium evaporation refrigerator. A superconducting Helmholtz coil provided a uniform 4.8 Tesla field within the active target volume. The radicals created by the radiation doping acted as paramagnetic sites; these sites coupled $138 \mathrm{GHz}$ microwaves to the nuclear spins to drive the hyperfine transition that polarized the target. The target polarization was reversed every few few hours by adjusting the microwave frequency. The polarization was measured using NMR coils imbedded in the cell and calibrated to the calculated thermal-equilibrium signal at $1.6^{\circ} \mathrm{K}$ with the microwaves turned off. The target polarization typically decayed from $75 \%$ to $50 \%$ during several hours of exposure to the beam. The polarization could then be restored by annealing the $\mathrm{NH}_{3}$ at $80^{\circ} \mathrm{K}$. The target material was replaced after about 10 annealing cycles.

\section{The Polarized Electron Beams}

Polarized electrons for E142 were generated by photoemission from an AlGaAs photocathode illuminated by circularly polarized laser light tuned to the AlGaAs band gap energy. [9] The polarized source delivered about $10^{11}$ electrons within a $1 \mu$ s pulse at $120 \mathrm{~Hz}$. The electron helicity was reversed randomly on a pulse-to-pulse basis by reversing the circular polarization of the laser. The charge of the beam pulse was measured to a relative error of $10^{-4}$ with two calibrated toroids upstream of the target; no correlation between the charge and electron helicity for the 
beam pulse was observed.

A single-arm Møller polarimeter measured the cross section asymmetry between opposite helicity electrons that Møller scattered off of a magnetized target foil. The beam electron polarization $P_{e}$ was stable during the experiment with an average value of $38.8 \pm 1.6 \%$; the uncertainty is dominated by the measurement of the target foil magnetization.

Experiment E143 took advantage of the recently discovered high-polarization strained GaAs photocathodes. [10] Beam was delivered at the lower intensity of $\sim 3 \cdot 10^{9}$ electrons per pulse and rastered over the active target area. Both a single arm Møller polarimeter and a double arm coincidence Møller polarimeter instrumented with lead glass blocks measured the electron beam polarization. The two polarimeters agreed to within $1 \%$. On average, $P_{e}=83 \pm 3 \%$ for E143.

\section{Spectrometers}

Each spectrometer was designed in a reverse bend configuration using a pair of dipole magnets. [11] Photon background could then be suppressed by blocking any direct line of sight between the detector elements and the target. The momentum acceptance for both spectrometers had a low momentum cutoff at $6 \mathrm{GeV} / \mathrm{c}$ and was roughly constant over the kinematically allowed momentum range above $8 \mathrm{GeV} / \mathrm{c}$.

Electrons were distinguished from pions using pulse height information from two Čerenkov detectors operating at pion threshholds of 9 and $13 \mathrm{GeV}$. The $10^{-4}$ duty factor of the SLAC beam required the experiment to run at beam pulse intensities high enough to scatter several charged particles into the detector during the $1 \mu$ s beam spill; it was therefore necessary to instrument the Čerenekov detectors with multi-hit TDCs to provide nanosecond timing information for each event. Tracking was provided by hodoscope planes built from overlapping $3 \mathrm{~cm}$ wide scintillator strips that were also instrumented with multi-hit TDCs. The momentum resolution $\Delta E^{\prime} / E^{\prime}$ was $3 \%$ on average, and the momentum was calibrated to NMR measurements of the spectrometer magnets. The electron energy was measured with a 24 radiation length lead glass shower counter built from 200 lead glass blocks in a fly's eye arrangement. In addition to the energy measurement, the shower counter provided electron identification 
independently of the Čerenkov detectors. The shower energy resolution was typically $15 \% / \sqrt{E^{\prime}(\mathrm{GeV})}$ and calibrated by the magnet NMR measurements through the hodoscope momentum measurement.

\section{E. Data Analysis}

The measured asymmetry $A_{\|}$is derived from the counting rate asymmetry: $P_{T} P_{e} f A_{\|}=[N(\uparrow \Uparrow)-N(\downarrow \Uparrow)] /[N(\uparrow \uparrow$ )$+N(\downarrow \uparrow)]$, where the counting rates have been normalized to the incident beam charge and corrected for experimental deadtime. An analogous expression is used to extract $A_{\perp}$. The dilution factor $f$ represents the fraction of electrons that scatter from the polarized neutron in the E142 target or proton in the E143 target. Typically $f \sim 0.11 \pm 0.02$ for E142 and $f \sim 0.15$ with a relative error of $3 \%$ for E143. The shower counter position and energy determined both $x$ and $Q^{2}$ for the event. $A_{\|}$and $A_{\perp}$ are combined with a global fit to $R$ and $F_{1}$ from SLAC deep inelastic data [12] to extract $g_{1}(x)$. No significant $Q^{2}$ dependence for the quantity $g_{1}(x) / F_{1}(x)$ was observed in either experiment E142 or experiment E143. The results from both spectrometers were averaged together and radiative corrections applied $[13,14]$ to obtain $\int_{0.033}^{0.6} g_{1}^{n}(x) d x=-0.019 \pm 0.007$ (stat.) \pm 0.006 (syst.) at an average $Q^{2}$ of $2(\mathrm{GeV} / \mathrm{c})^{2}$ for E142 and $\int_{0.027}^{0.8} g_{1}^{p}(x) d x=0.120 \pm 0.004$ (stat.) \pm 0.009 (syst.) at an average $Q^{2}$ of $3(\mathrm{GeV} / \mathrm{c})^{2}$ for E143. In order to determine $\int_{0}^{1} g_{1}(x) d x$ it is necessary to extrapolate $g_{1}(x)$ over the unmeasured $x$ range. For the low $x$ region a Regge parametrization $g_{1}(x) \sim x^{\alpha}$ was used with $\alpha=0.2$ for E142 and $\alpha=0$ for E143. The E143 data was extrapolated into the high $x$ region by assuming the dependence $g_{1}^{p}(x) \sim(1-x)^{3}$ as $x \rightarrow 1$. For experiment E142 the asymmetry $A_{1} \simeq g_{1} / F_{1}$ for virtual photon-nucleon scattering was extrapolated to 1 as $x \rightarrow 1$. After extrapolation over the unmeasured $x$ range the integrals become $\int_{0}^{1} g_{1}^{n}(x) d x=-0.022 \pm 0.011$ for E142 and $\int_{0}^{1} g_{1}^{p}(x) d x=0.129 \pm 0.011$ for E143.

\section{CONCLUSIONS}

We may combine the E142 measurement with the preliminary E143 measurement to obtain $\int_{0}^{1}\left[g_{1}^{p}(x)-g_{1}^{n}(x)\right] d x=$ $0.151 \pm 0.015$ at $Q^{2}=3(\mathrm{GeV} / \mathrm{c})^{2}$. This is in satisfactory agreement with the Bjorken sum rule prediction of $0.171 \pm 0.008$, where we have used 

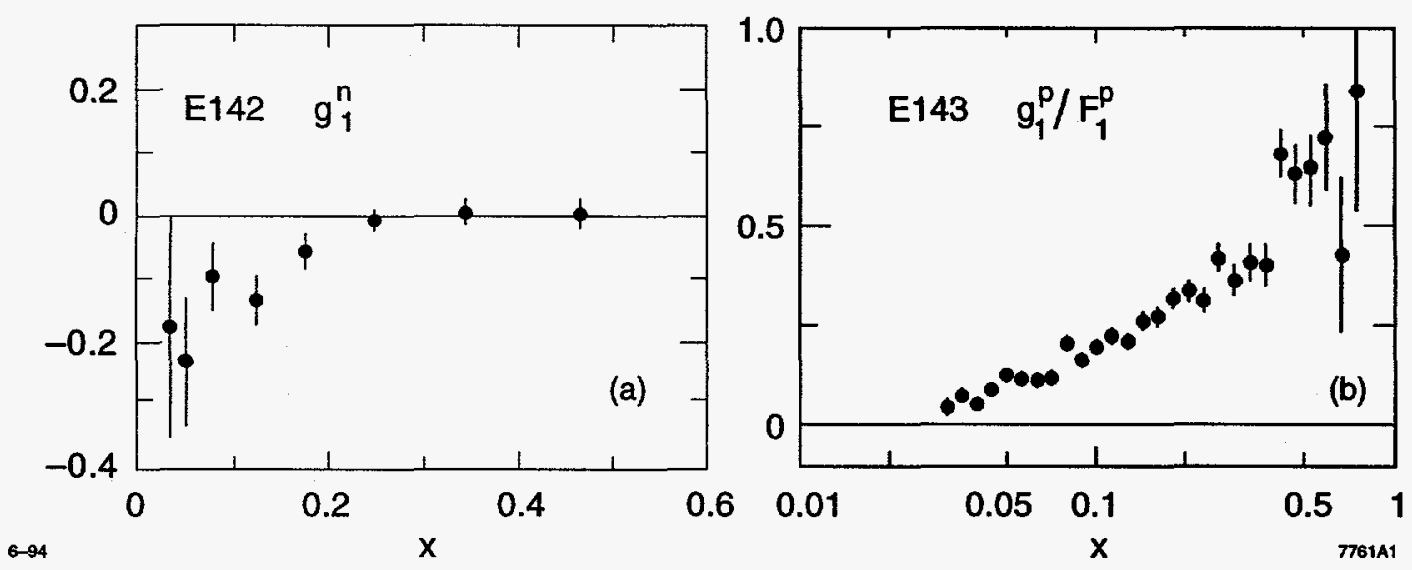

Fig. 1. (a) E142 data for $g_{1}^{n}(x)$. (b) E143 data for $g_{1}^{p}(x) / F_{1}^{p}(x)$.

$\alpha_{s}=0.35 \pm 0.05$ at $Q^{2}=3(\mathrm{GeV} / \mathrm{c})^{2}$. Using $\int_{0}^{1} g_{1}^{n}(x) d x$ from E142 with [15] $g_{A} / g_{V}=1.257 \pm 0.003, g_{A}^{\Sigma} / g_{V}^{\Sigma}=$ $-0.34 \pm 0.02$, and $\alpha_{s}=0.40 \pm 0.05$ we find $\Delta u=0.87 \pm 0.04, \Delta d=-0.39 \pm 0.04$, and $\Delta s=-0.05 \pm 0.04$. Using $\int_{0}^{1} g_{1}^{p}(x) d x$ from E143 with $\alpha_{s}=0.35 \pm 0.05$ we obtain $\Delta u=0.82 \pm 0.04, \Delta d=-0.44 \pm 0.04$, and $\Delta s=-0.10 \pm 0.04$. Summing the quark helicities, we estimate the total contribution of the quarks to the nucleon spin to be $0.44 \pm 0.10$ from E142 and $0.29 \pm 0.10$ from E143.

[1] J.D. Bjorken. Phys. Rev. 148 (1966) 1467; Phys. Rev. D1 (1970) 1376.

[2] S.A. Larin and and J.A.M. Vermaseren. Phys. Lett. B259 (1991) 345.

[3] S.A. Larin CERN-TH 7208/94 (1994).

[4] P.L. Anthony et al. Phys. Rev. Lett. 71 (1993) 959.

[5] K.Abe et al. SLAC-PUB-6087 (1994).

[6] R.M. Woloshyn. Nucl. Phys. 496A (1989) 749.

[7] T.E. Chupp et al. Phys. Rev. C45 (1992) 915; C36 (1987) 2244.

[8] D.G. Crabb et al. Phys. Rev. Lett. 64 (1990) 2627; W.Meyer et al. Nucl. Instrum. Meth. 215 (1983) 65.

[9] T. Maruyama et al. J. Appl. Phys. 73 (1993) 5189.

[10] T. Maruyama, E. Garwin, R. Prepost, and G. Zapalac Phys. Rev B46 (1992) 4261.

[11] G.G. Petratos et al. SLAC-PUB-5678 (1991).

[12] L.W. Whitlow et al. Phys. Lett B250 (1990) 193; B282 (1992) 475.

[13] L.W. Mo and Y.S. Tsai Rev. Mod. Phys. 41 (1969) 205.

[14] T.V. Kukhto and N.M. Shumeiko Nucl. Phys. B219 (1983) 412.

[15] Particle Data Group Phys. Rev. D45 (1992) S1. 